\title{
Application of simulation models for the optimization of business processes
}

Roman Jašek, Michal Sedláček, Bronislav Chramcov, and Jiř́ Dvořák

Citation: AIP Conference Proceedings 1738, 120028 (2016); doi: 10.1063/1.4951911

View online: http://dx.doi.org/10.1063/1.4951911

View Table of Contents: http://aip.scitation.org/toc/apc/1738/1

Published by the American Institute of Physics 


\title{
Application Of Simulation Models For The Optimization Of Business Processes
}

\author{
Roman Jašek, Michal Sedláček, Bronislav Chramcov and Jiř́ Dvořák \\ Tomas Bata University in Zlin \\ Nad Stranemi 4511, 76005 Zlin, Czech republic \\ jasek@fai.utb.cz,michal.sedlacek@email.cz,dvorakj@aconte.cz
}

\begin{abstract}
The paper deals with the applications of modeling and simulation tools in the optimization of business processes, especially in solving an optimization of signal flow in security company. As a modeling tool was selected Simul8 software that is used to process modeling based on discrete event simulation and which enables the creation of a visual model of production and distribution processes.
\end{abstract}

Keywords: modeling and simulation of processes, SIMUL8, model of logistic processes, linking theory with practice, business processes, generate random numbers.

PACS: 07.05.Tp, 05.45.Pq.

\section{INTRODUCTION}

Modeling and simulation in recent years became an indispensable tool in streamlining production systems, human activities and also in areas such as services. Computer simulation can be considered as an important facilitator of problems solution requiring the use of such methods and means of support, which allows a comprehensive approach to designing systems and quickly tried various solutions and minimize the risk of erroneous decisions. One of the basic cybernetic approaches exploring, analyzing, designing and planning systems are in modeling and models. Modeling is an essential element model $[1,3]$. The modeling and simulation is the technical term model is applied to the analogy between two systems (Fig. 1).

System - is purposefully simplified part of the real world, which is under review

Model - a simplified representation system using mathematical equations, graphs, verbal rules or images.

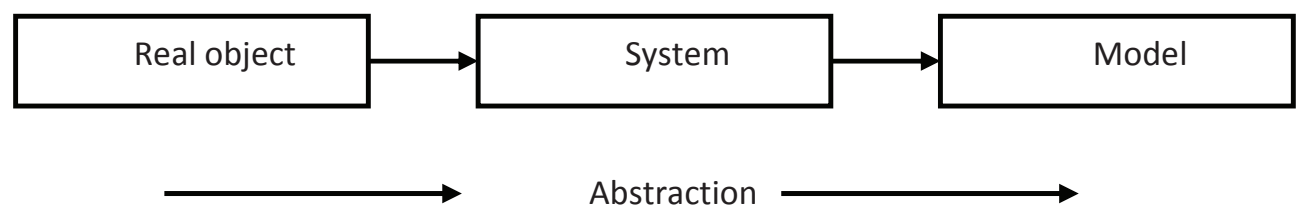

FIGURE 1. The relationship between real objects, systems and models

Simulation is the process of designing a model of a real system and the realization of experiments with this model, in order to understand the behavior of the system. Using the simulation we gain knowledge and information about the examination system and its elements.

\section{COMPUTER SIMULATIONS}

Target of creating the simulation model $[2,3]$ is to construct a description of the system, to have an idea about its function, to estimates its future behavior, to find an optimal solution to the problem and to work towards the proposed new structure of the system. The simulation model offers a solution of the problem, but will allow decision-makers to change the settings and variables that can influence even in the real object. After testing the models we can choose the most appropriate combination of settings, which subsequently implemented in the real environment. Nowadays, the analysis of complex processes and systems mainly used computer simulations. Its main advantage is to test the model in a virtual environment without affecting the real system under examination. Therefore, those simulation methods are used for testing hazardous, time or financially demanding projects. Simulations eliminates the shortcomings of analytical methods, but is also more time-consuming (for example model building, model testing, planning and

\footnotetext{
International Conference of Numerical Analysis and Applied Mathematics 2015 (ICNAAM 2015)

AIP Conf. Proc. 1738, 120028-1-120028-4; doi: 10.1063/1.4951911

Published by AIP Publishing. 978-0-7354-1392-4/\$30.00
} 
execution of experiments) as well, as in the preparation of input data. In the way of creating the simulation models it is possible to use appropriate simulation software

\section{RANDOM NUMBERS IN SIMULATION MODELS}

When creating simulation models we will meet the situation when we need to reach a certain randomness processes such as demand, orders approaching, the duration of a process. We are talking about the so-called random number generation [3]. Random numbers are independent values even distribution on the open interval $(0,1)$, which is denoted $\mathrm{R}(0,1)$. Equitable distribution is the simplest continuous distribution, but it is used for generating values of other distributions. Basic features even distribution of the probability density, distribution function, mean and variance.

\section{Continuous distribution}

Distribution function for a continuous distribution assigns to every real number $\mathrm{x}$ the probability that a random variable takes the value of less than or equal to $\mathrm{x}, F(x)=P(X \leq x)$. It is a non-decreasing function for which it is valid $0 \leq F(x) \leq 1$.

The probability density is for continuous random variables expressed as a function

$$
f(x)=\frac{d F(x)}{d x} \text { for all real } \mathrm{x}, \text { wherein } f(x) \geq 0 \text { and } \int_{-\infty}^{\infty} f(x) d x=1
$$

To generate random numbers in business process solutions mainly use normal, Poisson and exponential distribution. Such a random variable is reflected at random intervals generate input entities, which in turn passes the simulation model. For the development of computer simulations are used primarily arithmetic generators. These works by the next number is calculated with the previous figures. Because a random number will finalize the calculation paradoxically, we are talking about so-called pseudo-random numbers. There is a certain probability periodicity, but with today's level of computer technology, we can work seamlessly with the period length $2^{64}$.

\section{APPLICATION OF SIMULATION MODELS IN BUSINESS PROCESSES}

At the business process solutions and the subsequent creation of simulation models we use SIMUL8 simulation program $(1,2,9)$. It is a product of the same name SIMUL8 Corporation and is intended for the creation of discrete simulation mainly business processes. SIMUL8 enables you to create a visual model of monitoring a system where the course simulating display animation format, which is easy to check the correctness and functionality of the model. Due to the simplicity and clarity it is possible to construct the model is relatively quick and easy. Visual representation is used from the outset as a suitable means of communication between the complainant and the customer model. The program SIMUL8 includes the following essential elements and objects:

- Work Item (work item entity) - is an element which serves to create a dynamic element that moves through the model. Entities can enter the system, transform itself, using defined resources and the end to leave the system.

- Work Entry Point (input) - is the point of entry into the system entities.

- Work Center (action, activity) - entity operating under passes. Based on human judgment or real data, each activity associated with certain resources.

- Storage Bin (stack, queue) - an element which shows the accumulation of the models entities.

- Work Exit Point (output) - In the longitudinal represents an object through which the entity leaves the system.

- Resource (source) - object that represents the limited capacity of the materials, intermediate products, people that are used by entities in the activities.

- Route (path) - connections between elements of the model. Path represents the connection of activities and entities determined by the movement system.

\section{Simulation Model of the Security System}

An example of a particular use of a simulation model in dealing with business process optimization is to optimize information flow within the company KEN SECURITY s.r.o., which provides services in the field of property and personal security. Services are provided through physical surveillance and electronic security [3]. 


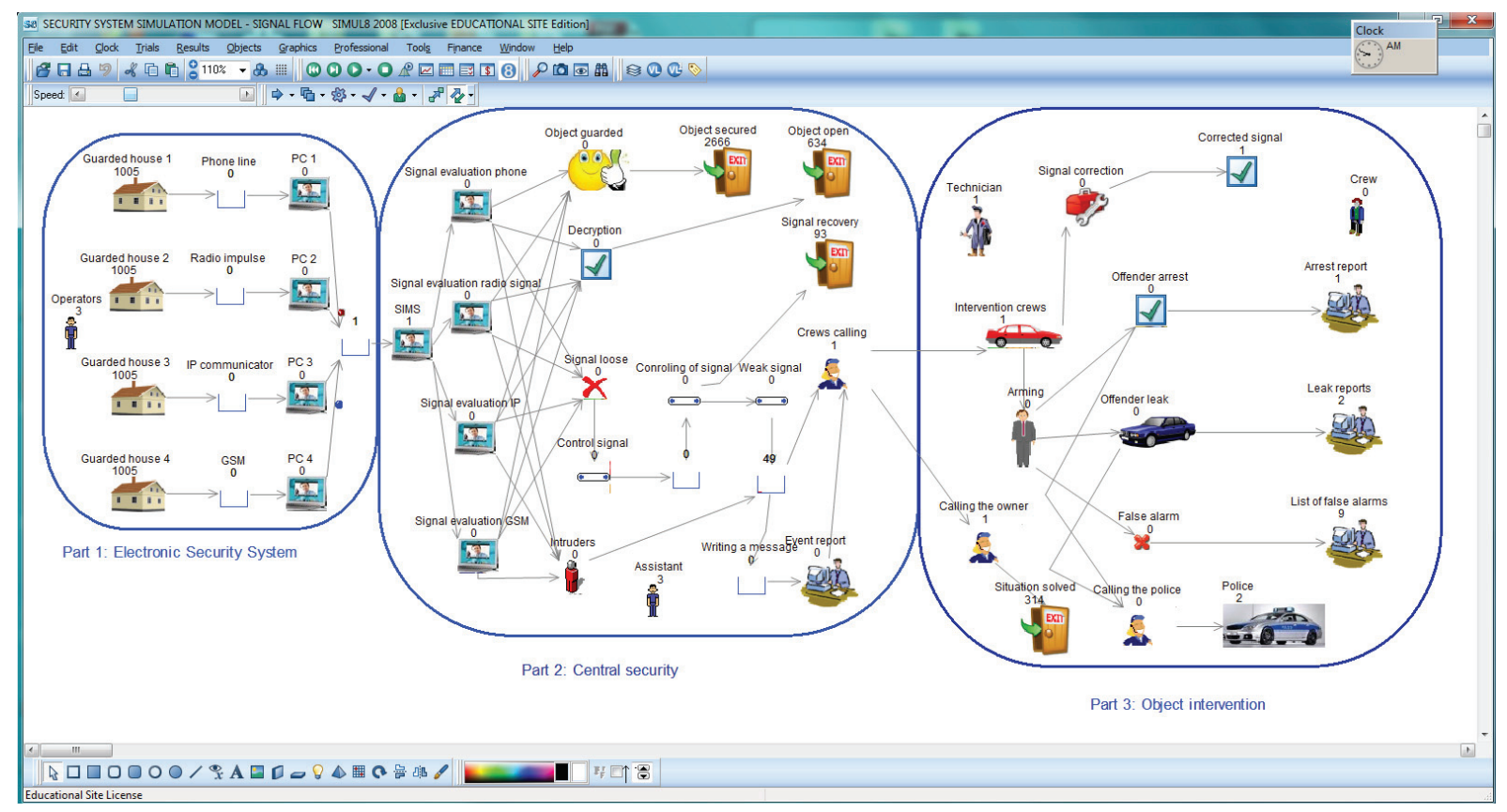

FIGURE 2. Simulation model of the security system [3]

In solving the problem, it is necessary to analyze the weaknesses in the security of electronic information flow and identify the causes of the decreasing clients who go to the competition. These causes will try to eliminate as part of the optimization of the entire company's information system and propose an improved flow of information through the introduction of modern technology. Everything with regard to maintaining optimal costs so that the company has achieved the same or better results as the competition and provide the right service at the right quality, at the right price to the right customer at the right time, quantity and place.

\section{Setting Parameters of the Simulation Model}

Appropriate tool to make a simulation model is the SIMUL8 program that is capable map the logistics information system of electronic security provided by KEN SECURITY s.r.o. Setting the individual parts of the simulation model (Fig.2) results primarily based from the current statistics in the firm and expert estimates, the company's employees. The simulation is set in seconds and logically divided into 3 main sections called Part 1, Part 2 and Part 3 . The signal monitoring runs in four buildings connected to the ARC for 8 hours. In the real state of ongoing monitoring 24 hours a day, but due to the possibilities of the program and SIMUL8 for better orientation is selected simulation period of 8 hours. Electronic signals from the objects are generated in the period of 10 seconds.

Security is based on the location of electronic security systems in buildings and connections to central security. The security system transmits a signal every 10 seconds over 4 connection systems, which are chosen primarily from the position of the object. The aim of the system can also prevent the penetration of unwanted persons into secure areas. Unauthorized entry of persons is detected with the aid of a motion detector or magnetic contact. Individual motions detectors deploy in the building transmit a signal to a central protection or owner of the property. The brain of the security system is alarm, which is connected via cable or wirelessly to a central protection.

In creating a simulation model we must have an exact idea of the flow of information (entities) in the system. Based on the initial analysis of the system we set the different elements of the simulation model. The basic setup includes time generating entities that enter into the system, time signal processing-unit, intervention times and work of human resources.

When we are setting a behavior of the system is necessary to take into account of the occurrence of random events, such as loss of signal, communication error, mistakes and human resources faults like random elements. Such random variables in simulation models we set the distribution function of time units primarily at entry points and work centers. Preferably we use Normal, Exponential and Poisson distribution. In the process of moving and concretization entities flow in the system we use labels, that matches the attributes of entities, under which work centers entity divided into directions (roots) or processed in time. 


\section{CONCLUSION}

Using of modeling and simulation programs, in the way of solving the business processes, has its own distinct place in terms of proper articulation of decision-making processes and simulation tools. Simulation experiments can reveal problems and complications that should occur only in normal operation, thus saving considerable costs, which we had to spend on their removal. In some cases, it is virtually impossible in practice to try various solutions due to the irreversible steps or a very expensive process. Simulation models are able to solve those situations by the much cheaper way. Modeling and simulation can be very powerful tool for optimizing business processes.

The aim of the presented simulation model is to highlight weaknesses in information flow and subsequently propose a new flow of information so that the company will ensure better service delivery and increase the number of clients and profit. Analysis and design of a new system of information flow is performed using a simulation programme called SIMUL8. Optimalized model is based primarily on the integration of modern technology, extension of tangible assets and human resources in the company. This change allows for the provision of quality services for the best price and keeps the company at the level of its competitors.

In this paper we wanted to outline the practical use of computer simulation models for solutions to optimize business processes. Simulation model that can help us get a glimpse of the behavior of the system and using appropriate experiments to analyze the bottlenecks of the process.

\section{ACKNOWLEDGMENTS}

The work was performed with financial support of research project NPU I No. MSMT-7778/2014 by the Ministry of Education of the Czech Republic and also by the European Regional Development Fund under the Project CEBIA-Tech No. CZ.1.05/2.1.00/03.0089 and Project No. 1.07/2.3.00/20.0035 for the development of human resources for science and research activities of Tomas Bata University in Zlin.

\section{REFERENCES}

1. Volna, E., Kotyrba, M., and Janosek, M. „Knowledge discovery in dynamic data using neural networks.“ In Kuinam J. Kim, K. J. (ed.) Information Science and Applications. Lecture Notes in Electrical Engineering (Book series), Springer Verlag Berlin Heidelberg, 2015, Volume 339, pp. 575-582. ISSN: 18761100. DOI: 10.1007/978-3-66246578-3_67.

2. Bucki, R., Chramcov B., Suchanek P. (2015). Heuristic algorithms for manufacturing and replacement strategies of the production system. Journal of Universal Computer Science 21 (4): 503-25, ISSN 0948-695X.

3. Chramcov B., Bucki R. Lean Manufacturing System Design Based on Computer Simulation: Case Study for Manufacturing of Automotive Engine Control Units, in Handbook of Research on Design and Management of Lean Production Systems, Vladimír Modrák and Pavol Semančo, Eds. Hershey, PA, USA: IGI Global, 2014, pp. 89-114. 